\title{
ESTIMACIÓN DEL COSTO DE REPARACIÓN DEL DAÑO AMBIENTAL EN EL SUELO DE CACAO EN LA PROVINCIA DE COTOPAXI-ECUADOR.
}

\section{ESTIMATION OF THE COST OF REPAIRING THE ENVIRONMENTAL DAMAGE IN THE COCOA SOIL IN THE PROVINCE OF COTOPAXI-ECUADOR.}

Sergio Leonardo Pino Peralta, Mgs.

Magíster en Economía Agrícola y Desarrollo Rural (Ecuador). Docente titular a tiempo completo de la Facultad de Ciencias Económicas de la Universidad de Guayaquil, Ecuador. sergio.poniop@ug.edu.ec

Alfonso Casanova Montero, PhD. Doctor en Ciencias Económicas (Cuba). Docente titular a tiempo completo de la Facultad de Ciencias Económicas de la Universidad de Guayaquil, Ecuador. alfonso.casanovam@ug.edu.ec

\section{ARTÍCULO DE INVESTIGACIÓN}

Recibido: 9 de noviembre de 2018.

Aceptado: 11 de enero de 2019.

\section{RESUMEN}

Ecuador es reconocido a nivel mundial por ser el mayor oferente de cacao fino de aroma (variedad arriba), cuya materia prima es muy demandada por la industria chocolatera, especialmente por países europeos, quienes la utilizan en la elaboración de los más finos chocolates "dark". El avance sostenido de la variedad CCN51 en la zona de esta investigación, está ocasionando problemas ambientales por el uso de agroquímicos para el control de enfermedades y plagas, deterioro de la flora y fauna, así como del suelo y fuentes de agua. El objetivo del presente artículo es valorar económicamente el daño ambiental provocado por la intervención antropocéntrica, por medio de la estimación del costo de reparación del recurso suelo en la zona afectada por la siembra del cacao CCN51. La metodología para estimar el daño ambiental en esta región fue de tipo longitudinal, mediante un modelo matemático que permitió calcular el costo de reparación del suelo entre los años 2011 y 2018. El estudio confirmó que existe un daño ambiental al suelo, por 
efecto del cambio de variedad en este período de análisis, calculado a un costo de USD $764,00 /$ ha. para reparar ese recurso. Por ello, se sugiere que los gestores de la política pública agropecuaria del Ecuador consideren estos resultados ambientales como aporte al sector cacaotero.

Palabras clave: cacao arriba, costo de reparación, daño ambiental, ecosistema, valoración económica.

\section{ABSTRACT}

Ecuador is recognized worldwide for being the largest supplier of fine aroma cocoa (variety Arriba), whose raw material is highly demanded by the chocolate industry, especially by European countries, who use it in the production of the finest chocolates "dark". The sustained advance of the CCN51 variety in the area of this research is causing environmental problems due to the use of agrochemicals for the control of diseases and pests, deterioration of the flora and fauna, as well as the soil and water sources. The objective of this article is to economically assess the environmental damage caused by anthropocentric intervention, by estimating the cost of repairing the soil resource in the area affected by the cocoa planting CCN51. The methodology for estimating environmental damage in this region was longitudinal, using a mathematical model that allowed calculating the cost of soil repair between 2011 and 2018. The study confirmed that there is environmental damage to the soil, due to the change of variety in this period of analysis, calculated at a cost of USD 764.00 / ha. to repair that resource. Therefore, it is suggested that the managers of the agricultural public policy of Ecuador consider these.

Keywords: arriba cocoa, repair cost, environmental damage, ecosystem, economic valuation.

\section{INTRODUCCIÓN}

El Ecuador es reconocido a nivel mundial por ser uno de los pocos países que produce un cacao con características especiales de sabor y aroma (cacao variedad arriba), materia prima que es altamente demandada por la industria chocolatera, especialmente por países europeos, quienes la utilizan en la elaboración de los más finos chocolates "dark" o "gourmet". Un alto porcentaje de este tipo de cacao es producido por unos 60000 pequeños productores que tienen en promedio menos de 10 hectáreas, que como rasgo distintivo es 
la casi inexistente aplicación de insumos químicos para el manejo y control fitosanitario de la plantación.

Por otro lado, en el año 1965 se liberó una nueva variedad de cacao llamado "CCN51" (colección castro naranjal), que se diferencia con el cacao arriba por presentar un mayor rendimiento, mayor precocidad, resistencia a enfermedades endémicas del cacao (escoba de bruja y monilia), árbol de bajo tamaño que facilita la cosecha y sabor a chocolate sin la presencia de sabores florales. Sin embargo, su manejo está restringido al uso intensivo de agroquímicos, y sobre todo a la siembra en monocultivo en grandes extensiones de terreno. En el Ecuador no existen muchos estudios que hayan valorado el servicio ambiental de cultivos agrícolas, por ello con esta investigación se pretende encontrar el punto de inflexión que permita reorientar las políticas públicas del sector cacaotero ecuatoriano, demostrando que el costo de reparación del daño del bien ambiental suelo, por efecto de la reconversión productiva de cacao arriba (cultivado en forma tradicional) por el cacao CCN51 (monocultivo intensivo con uso de agroquímicos) puede resultar muy alto ya que, si bien se obtendría una mayor rentabilidad, en cambio, estaría perdiendo la posibilidad de mantener un ecosistema libre de agentes externos contaminantes al medio ambiente; así como la disminución de la fertilidad natural del suelo, contaminación de fuentes de agua para consumo humano y regadío; y, por último, la reducción de la biodiversidad (flora y fauna) que existe en las plantaciones de cacao arriba. En ese sentido, este estudio tuvo como objetivo, estimar el valor económico del daño ambiental del agroecosistema Cacao (Theobroma cacao L.) var. Arriba en la Provincia de Cotopaxi-Ecuador.

Casi siempre es complicado valorar las externalidades de un proyecto, y para ello, es importante medir e identificar esos impactos. Por otro lado, el rigor de los impactos ambientales, también están en relación con los problemas acumulados en el tiempo y en un determinado lugar, o la mezcla de las dos cosas. Así mismo, es preciso contar con datos que muchas veces son de pésima calidad y escasos, lo cual aumenta las dificultades para realizar una buena valoración económica (Dixon y Pagiola 1998).

Pengue (2009) analizando la evolución y situación de los nutrientes del suelo, como un indicador de pérdida de calidad ambiental, social y económica, establece la importancia del recurso suelo en actividades agrícolas, y su fuerte relación con la "agroculturización" exacerbada con el impulso de la "revolución verde"; señala entre otras aspectos ambientales, que los paquetes asociados con el herbicida glifosato, el cultivo de soja transgénica y la ganadería intensiva, generan una pérdida de nutrientes y degradación de 
suelos, destrucción de hábitats, aparición de nuevas plagas, enfermedades y malezas tolerantes y resistentes, sumados a la deforestación en varias ecorregiones. El estudio se centra en un análisis sobre la evolución y situación de los nutrientes del suelo, como un indicador de pérdida de calidad ambiental, social y económica.

Silva y Correa (2009) explican que la contaminación del suelo puede alterar la vida de los seres vivos, ya que es uno de los elementos más importantes del medio ambiente, no renovable, limitado, de larga recuperación e imprescindible para la vida misma de seres humanos y otras especies animales y vegetales. Además, es sustento de actividades económicas, especialmente la agricultura y ganadería, procesos donde se usan plaguicidas y demás insumos para la obtención de alimentos u otros productos (biocombustibles) que están deteriorando este recurso de manera acelerada. Por esta razón, dicen los autores que se deben crear políticas públicas que permitan la regulación en el uso del suelo para reducir el impacto negativo, a la vez que posibilite genera conciencia sobre la importancia de preservar los recursos del medio ambiente.

Los mismos autores, señalan en otro estudio que los contaminantes que causan un gran deterioro en el suelo son los plaguicidas, compuestos tóxicos que se usan indiscriminadamente para combatir plagas y enfermedades de los cultivos. Estas sustancias, bien pueden acumularse en el suelo, contaminar a través de la filtración en aguas subterráneas, afectar la biodiversidad de un ecosistema en particular, y en consecuencia dañar la cadena trófica, pudiéndose perder de forma irrecuperable los bienes y servicios entregados por el suelo.

El daño ambiental se refiere a cualquier actividad, omisión, acto o comportamiento de un ente público o privado, individual o sujeto jurídico, que modifique, altere, deteriore, transforme, reduzca o ponga en riesgo inminente, algún recurso del ambiente, que desequilibre un ecosistema. El daño ambiental puede ocurrir de manera fortuita 0 accidental causada por la propia naturaleza, no obstante, los mayores daños son provocados por la acción antropocéntrica que degrada en forma significativa y relevante el medio ambiente (Peña 2013).

Este daño puede tener cuatro características: manifestación, efectos, causas e involucrados. Según el mismo autor, se pueden tomar acciones preventivas y curativas, frente a un daño, y también se puede sumar una tercera, que es la de mitigación (Barrantes 
y Di Mare 2001). Los costos por daños ambientales no se pueden medir como parte del PIB, pero se evidencia en el bienestar de la sociedad (Osorio y Correa 2004).

Dixon y Pagiola (1998), manifiestan que cuando los beneficios de un impacto ambiental no pueden ser calculados en forma directa, se usa la información de costos para determinar con más exactitud el valor económico del daño ambiental. También se asume que los costos de reemplazo o restauración no deben ser superiores al valor económico del activo ambiental. Sin embargo, a veces puede resultar más caro reparar o reemplazar un activo original. Así, por ejemplo, en un cultivo laderable que ha sido erosionado se sugiere la construcción de terrazas para reducir o prevenir la erosión. Esta medida preventiva puede tener un costo superior al valor inicial del activo ambiental. De hecho, para reponer un daño ambiental, no solo hay que observar que la técnica sea la apropiada, sino que además sea eficiente.

\section{MATERIALES Y MÉTODOS}

El estudio se llevó a cabo en campos cacaoteros de la Comunidad "Unión de Organizaciones de Productores Agropecuarios de Moraspungo" (UOPAM) en la Parroquia Moraspungo, Cantón Pangua, Provincia de Cotopaxi, República del Ecuador, cuya ubicación Geodésica UTM es: 17 M 690100,00 m E, 9874715,00 m S, elevación 745 msnm (Fig. 1).

La investigación es de tipo descriptiva, y se espera que el nuevo conocimiento obtenido sirva de instrumento de toma de decisiones para conservar el ecosistema cacao arriba en la zona de estudio y todo el país. A través de indagaciones descriptivas se conoce cómo la pérdida recurrente y sistemática de la superficie dedicada tradicionalmente al cultivo de cacao arriba, va perdiendo terreno frente a la siembra de cacao CCN51 acompañado de un paquete tecnológico exigente en insumos externos y uso intensivo del recurso suelo. 


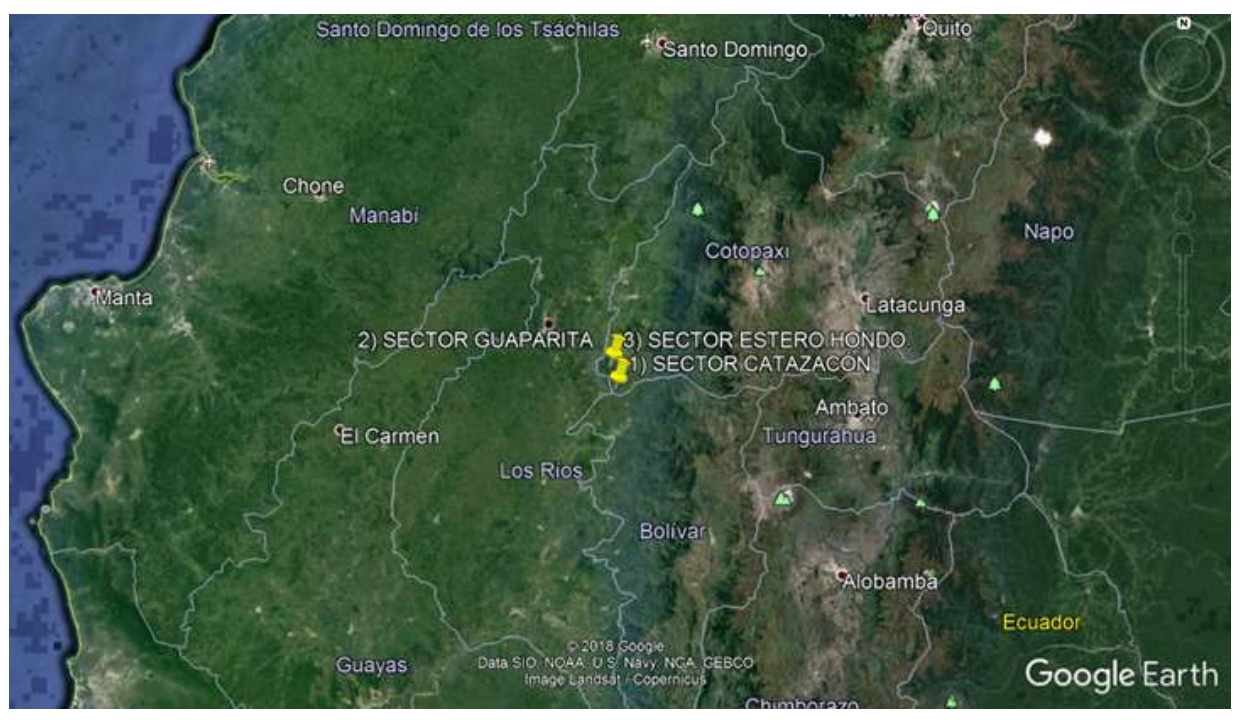

Figura 1. Ubicación satelital de la UOPAM.II Fuente: Elaboración propia.

También, el estudio es de tipo correlacional, pues mide el grado de relación que tienen las variables en estudio, pues una mayor aplicación de agroquímicos para la producción de cacao CCN51, supone un mayor daño ambiental en el suelo; además, de ser longitudinal, toda vez que los datos fueron tomados a través del tiempo, para realizar análisis comparativos entre períodos diferentes con el propósito de establecer el cambio ocurrido tanto en los niveles de rentabilidad financiera como en el daño ambiental ocurrido en el tiempo (Müggenburg y Pérez 2007).

Para valorar económicamente el daño ambiental, se estimaron todos los costos para reemplazar o reponer el bien ambiental afectado o deteriorado. (aspecto biofísico del suelo). Para una aplicación adecuada de este método de valoración, en este estudio se conoció la situación antes de que el bien se haya contaminado o dañado. En este sentido, se obtuvo información del estado físico - químico del suelo en la zona de estudio del año 2011, la cual se comparó con el estado actual del suelo (2018), lo que permitió contar con parámetros comparativos que determinaron cambios y en base a esos cambios, se valoraron los costos de reposición del recurso suelo en relación al estado anterior a su afectación (Peña,1989).

El costo de la reparación del bien ambiental hasta los niveles aceptables se determinó por la magnitud del daño ocasionado, las características del suelo, el tiempo de la recuperación y el área afectada. Gráficamente, el costo de recuperación (CR) es el área correspondiente 
bajo la curva $f(x)$ en el intervalo de tiempo $(0, T)$, donde $x$ es un vector de variables que explican la afectación biofísica del recurso natural (Barrantes y Di Mare 2001) (Fig. 2).

$C R=\int_{0}^{T} f(x) d x$

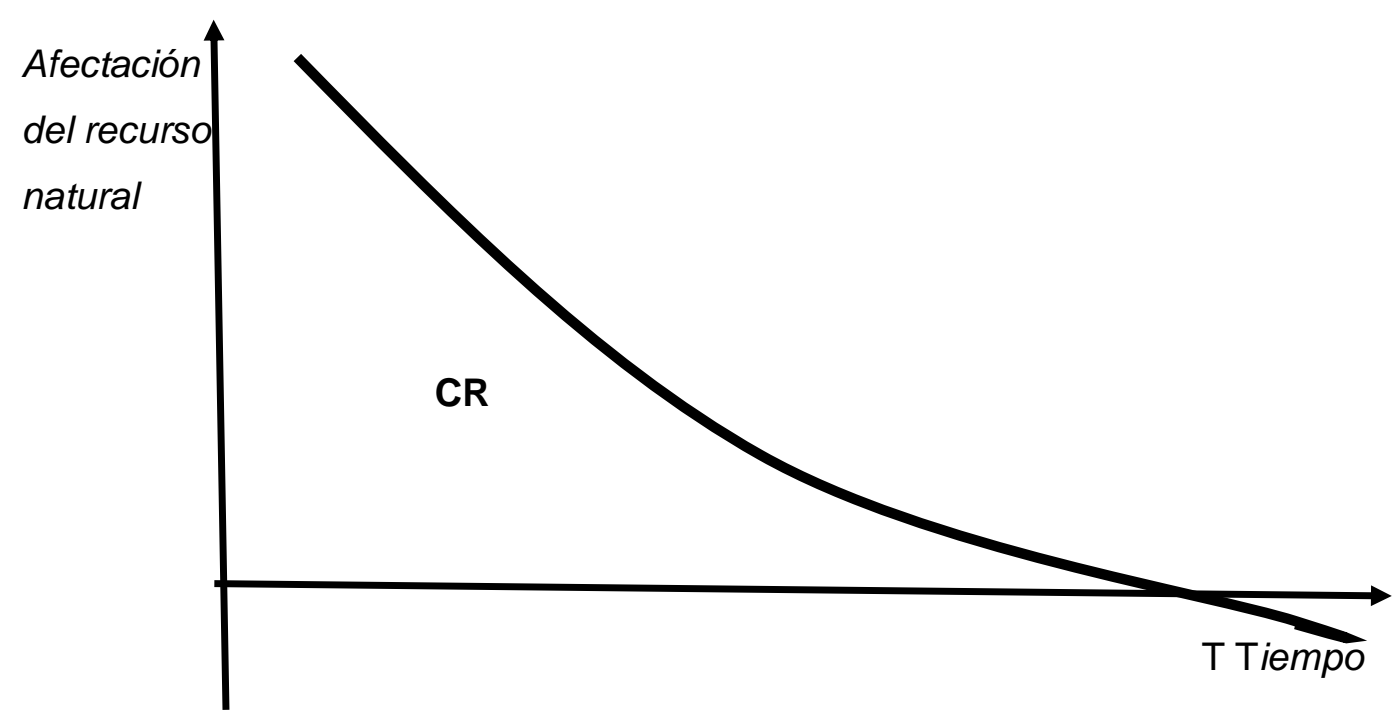

Figura 2. Costo de recuperación del recurso natural afectado.

Fuente: Elaboración propia.

$$
\begin{aligned}
& C R=\sum_{t=0}^{T} \sum_{j=1}^{n} \sum_{i=1}^{m} p_{i} q_{t j i}(1+r)^{-t} \\
& \\
& C R=\left(P_{1} Q_{011}+P_{2} Q_{012}+P_{3} Q_{013}\right)(1+0.12)^{0} \\
&+\left(P_{1} Q_{111}+P_{2} Q_{112}+P_{3} Q_{113}\right)(1+0.12)^{-1} \\
&+\left(P_{1} Q_{211}+P_{2} Q_{212}+P_{3} Q_{213}\right)(1+0.12)^{-2} \\
&+\left(P_{1} Q_{311}+P_{2} Q_{312}+P_{3} Q_{313}\right)(1+0.12)^{-3} \\
&+\left(P_{1} Q_{411}+P_{2} Q_{412}+P_{3} Q_{413}\right)(1+0.12)^{-4}
\end{aligned}
$$

Dónde:

CR: $\quad$ Costo de restauración biofísica del suelo afectado por aplicación de agroquímicos (c/unidad del factor)

T: $\quad \operatorname{Max}\left\{t_{-} j / j\right.$ es el recurso natural y $\left.j=1,2, \ldots, n\right\}$

$\boldsymbol{p}_{-} \boldsymbol{i}$ : $\quad$ Precio del insumo $i$ usado en la restauración del suelo (c/unidad del insumo)

$\boldsymbol{q}_{-} \boldsymbol{i} \boldsymbol{j}$ : $\quad$ Cantidad del insumo $i$ usada en la restauración del recurso natural (suelo) $j$ (unidades del insumo)

$r$ : $\quad$ Tasa de descuento para actualizar los valores en el tiempo (12\%) 

$\boldsymbol{t}: \quad$ Tiempo (años)
T: Tiempo total requerido para la restauración del daño causado, determinado por el estado de conservación de los recursos naturales alterados. $(T=5)$
m: Insumos requeridos en la restauración del recurso natural (suelo) $i(m=2)$
n: Recurso natural afectado (suelo) por acción humana $(\mathrm{n}=1)$

Para obtener el requerimiento interno de los elementos químicos faltantes en el suelo (nitrógeno y fósforo), se calculó con la siguiente fórmula:

$$
R N=\frac{N N-(20 \times 3,9 M O \times 1,08 D A)}{0,6}
$$

Dónde:

$$
\begin{aligned}
& \mathrm{RN}=\text { Requerimiento nutricional } \\
& \mathrm{NN}=\text { Necesidades nutricionales }(151 \mathrm{~kg} / \mathrm{ha}) \\
& \mathrm{MO}=\text { Materia orgánica } \\
& \mathrm{DA}=\text { Densidad aparente }
\end{aligned}
$$

\section{RESULTADOS Y DISCUSIÓN}

Para determinar el daño del bien ambiental suelo, se consideraron los resultados de los análisis de suelos realizados en el mes de abril de 2011, en el marco de la ejecución del proyecto de investigación denominado "Investigación agro-socioeconómica de la cadena de valor del cacao Fino de Aroma con Organizaciones de Pequeños Productores en las estribaciones de cordillera de las Provincias de Los Ríos, Cotopaxi y Bolívar-Ecuador", que fue contrastarlo con los análisis de suelos realizados en junio de 2018 en lotes de cacao de productores de la UOPAM.

Una consideración importante es que las muestras de suelos, tanto en el 2011 como en el 2018 fueron tomadas en las parcelas donde los productores tenían sembrado cacao arriba; y en la actualidad han migrado a cacao CCN51, procedimiento que permitió realizar el análisis físico - químico comparativo en épocas y tipos de cacao diferentes. Las muestras tomadas mediante el método de zigzag a una profundidad de $20 \mathrm{~cm}$ donde está concentrada el $80 \%$ del sistema radical de la planta de cacao En este contexto, los resultados de los análisis comparativos entre estos años, desde el punto de vista ambiental y conservacionista evidencia que en las parcelas donde se produjo 
la reconversión de cacao arriba por cacao CCN51 se encontraron diferencias sustanciales (Tabla 1 ).

Tabla 1. Análisis físico-químico del suelo (2011 - 2018) en la zona de Moraspungo, Provincia Cotopaxi, 2018.

\begin{tabular}{|c|c|c|c|c|c|c|c|c|c|c|c|c|c|c|}
\hline Muestra & elementos & $\mathrm{pH}$ & $\begin{array}{l}\mathbf{N} \\
\%\end{array}$ & $\begin{array}{c}\mathrm{P} \\
\mathrm{ppm}\end{array}$ & $\begin{array}{c}\mathrm{S} \\
\mathrm{ppm}\end{array}$ & $\begin{array}{c}\mathrm{K} \\
\mathrm{meq}\end{array}$ & $\begin{array}{c}\mathrm{K} \\
\mathrm{ppm}\end{array}$ & \multicolumn{2}{|c|}{ meq/100ml } & $\mathrm{Cu}$ & $\mathrm{Fe}$ & $\begin{array}{l}\mathrm{Mn} \\
\mathrm{ppm}\end{array}$ & $\mathrm{Zn}$ & B \\
\hline AÑO 2011 & PROMEDIO & 6,02 & 0,18 & 14,00 & 100 & 0,26 & 101,40 & 13,20 & 2,10 & 6,50 & 194,40 & 5,80 & 8,40 & 0,70 \\
\hline AÑO 2018 & PROMEDIO & $6,00^{\prime}$ & $0,17^{\prime}$ & $6,95^{\prime}$ & $37^{\prime \prime}$ & 0,61 & $237,90^{\prime \prime}$ & $18,40^{\prime}$ & 5,85 & 2,25 & $116,35^{\prime}$ & $44,00^{\prime}$ & $11,75^{\prime}$ & 0,48 \\
\hline \multirow{2}{*}{\multicolumn{2}{|c|}{$\begin{array}{l}\text { Rango mínimo } \\
\text { Rango máximo }\end{array}$}} & & 31 & 8 & 4,0 & 0,20 & 78,0 & 5,10 & 1,70 & 1,1 & 20,0 & 5,1 & 3,1 & 0,20 \\
\hline & & & 40 & 14 & 19,0 & 0,38 & 148,2 & 8,90 & 2,30 & 4,0 & 40,0 & 15,0 & 7,0 & 0,49 \\
\hline \multirow[t]{2}{*}{ Muestra } & profundidad & $\mathrm{Fe}$ & $\mathrm{Ca}$ & $\mathrm{Mg}$ & $\mathrm{Ca}+\mathrm{Mg}$ & S Bases & CICE & MO & $\mathrm{DA}$ & & Textura & & \multirow{2}{*}{\multicolumn{2}{|c|}{ Clase Textural }} \\
\hline & & $\mathrm{Mn}$ & $\mathrm{Mg}$ & $\mathrm{K}$ & $\mathrm{K}$ & \multicolumn{2}{|c|}{$\mathrm{meq} / 100 \mathrm{ml}$} & $\%$ & $\mathrm{Gr} / \mathrm{cm} 3$ & Arena & Arcilla & Limo & & \\
\hline AÑO 2011 & $20 \mathrm{Cm}$ & 33,52 & 6,29 & 8,08 & 58,85 & 15,56 & 35,40 & 3,90 & 1,08 & 40 & 49 & 11 & \multicolumn{2}{|c|}{ Franco } \\
\hline AÑO 2018 & $20 \mathrm{Cm}$ & 1,82 & 2,83 & 12,22 & 46,83 & 30,13 & 30,90 & 1,90 & 1,10 & 27 & 15 & 58 & \multicolumn{2}{|c|}{ Franco - Limoso } \\
\hline \multicolumn{2}{|c|}{ Rango mínimo } & 1,00 & 3,50 & 8,00 & 20,00 & & & 2,00 & & & & & & \\
\hline \multicolumn{2}{|c|}{ Rango máximo } & 3,00 & 4,00 & 15,00 & 38,00 & & & 4,00 & & & & & & \\
\hline
\end{tabular}

Fuente: Elaboración propia.

No hubo variación significativa del elemento $\mathrm{N}$, pero el contenido de materia orgánica disminuye ostensiblemente de 3,9 en el 2011 a 1,9 en el año 2018. La materia orgánica mejora las propiedades físicas y químicas del suelo elemento clave en la aportación de Nitrógeno. Algunos elementos disminuyeron drásticamente su concentración, como el $\mathrm{P}$ $(50 \%)$, S $(37 \%)$, Cu (63\%), B (45\%) y Fe (42\%); mientras que otros aumentaron significativamente, tal es el caso del K (160\%), Ca (40\%), Mg (150\%), Zn (40\%) y Mn (800\%).

En cuanto a las relaciones de cationes, el análisis comparativo fue el siguiente:

- Hierro (Fe) / Manganeso (Mn): bajó drásticamente de 35,52 ppm 2,58ppm.

- Calcio (Ca) v/ Magnesio (Mg): está dentro de los parámetros normales con 3,29 $\mathrm{meq} / 100 \mathrm{ml}$

- Magnesio (Mg) v/ Potasio (K): Subió a 9,50 meq/100ml.

- Calcio (Ca) + Magnesio $(\mathrm{Mg})$ / Potasio (K): disminuyó 39,51 meq/100ml 
- Suma de bases: se incrementó a 25 meq/100ml.

- La capacidad de intercambio catiónico: disminuyo a 25,45.

- La materia orgánica: disminuyó de 3,9\% a 1,9\% debido a la menor emisión de hojas del Cacao CCN-51.

- Densidad aparente: se mantiene igual.

- La Capacidad de intercambio catiónico: disminuyó de 35,40 a 25,45 meq/100 ml.

- Clase textural franco limoso: suelo adecuado para el cultivo de cacao.

En el transcurso de 7 años ha existido una ligera variación en el contenido nutricional del suelo. Lo más representativo es la disminución de la materia orgánica que obliga a realizar una aplicación adicional de Nitrógeno elemento clave para el crecimiento y desarrollo de la planta. El contenido de Fosforo disminuyó, elemento esencial para el desarrollo de las raíces por ende en la absorción de nutrientes.

En cuanto al nitrógeno del suelo se refiere, si consideró el requerimiento de cacao para una población de 1.100 plantas/ha., que es de $151 \mathrm{Kg}$ de nitrógeno/ha. Por lo tanto, el requerimiento interno de nitrógeno para el año 2011 se calculó de la siguiente manera: $R N$ $=151 \mathrm{Kg}-(20 \times 3,9 \mathrm{MO} \times 1,08 \mathrm{DA})=66,76$ dividido para una eficiencia de $60 \%$, el resultado es de $111,27 \mathrm{~kg}$ de Nitrógeno.

Para el año 2018, el RN = $151 \mathrm{~kg}-(20 \times 1,9 \mathrm{MO} \times 1,10 \mathrm{DA})=109,20 \mathrm{~kg}$ de $\mathrm{N}$ dividido para una eficiencia de 60\%, el resultado es de $182 \mathrm{~kg}$ de Nitrógeno. La diferencia entre $182 \mathrm{~kg}$ (año 2018) y 111,27 kg de N (año 2011), es de 70,73 kg la pérdida de $\mathrm{N}$ en 7 años. Esta cantidad al ser transformada en urea $(46 \% \mathrm{~N})$, dio $153,76 \mathrm{~kg}$, es decir aproximadamente 3,07 sacos de urea de $50 \mathrm{~kg}$ que deberán ser incorporadas al suelo por al menos cinco años para recuperar el nivel de nitrógeno que se tenía en el año 2011. El precio de mercado de la urea en la zona de Moraspungo es de USD 19,00/saco.

Un segundo elemento químico que se encontró con marcadas diferencias con respecto al año 2011 fue el fósforo. Se considera que el requerimiento de cacao con una población de 
1.100 plantas/ha., es de $38 \mathrm{~kg}$ de fósforo $(\mathrm{P})$ puro, y usando un factor conversión de 2,29 equivalen a $87,02 \mathrm{~kg}$ de $\mathrm{P} 2 \mathrm{O} 5 / \mathrm{ha}$. Usando la misma ecuación, el requerimiento de fósforo en el año 2011 fue de: 87,02 kg - (14 ppm x 3,435 x DA 1,08) = 35,08 dividido para una eficiencia de $30 \%$, el resultado es de116,9 $\mathrm{kg}$ de fósforo.

En el año 2018, haciendo los mismos cálculos, el requerimiento del cultivo es de: $87,02 \mathrm{~kg}$ - $(6,95$ ppm x 3,435 x DA 1,05) =61,95 dividido para una eficiencia de $30 \%$, el resultado es de $206,5 \mathrm{~kg}$ de fósforo. La diferencia entre los dos años analizados es de 89,6 kg de P2O5/ha, que transformados a Fosfato Di-amónico (DAP) al 46\%, da 194,8 kg DAP/ha; o, 3,9 sacos de $50 \mathrm{~kg}$ de18-46-00/ha. El precio de mercado de este insumo es de USD 30 /saco.

Para la aplicación de estos dos insumos faltantes en el suelo (urea y fosfato di-amónico), se requieren al menos dos jornales al año, de la siguiente forma: un jornal para la aplicación del $50 \%$ de los dos insumos a la entrada de las lluvias; y el otro jornal aplicará el $50 \%$ restante, a la salida de éstas. En los demás elementos químicos los cambios no son de mayor significancia o su variación es irrelevante para incorporar en la valoración económica del daño ambiental. Una vez obtenidos los valores faltantes de nitrógeno y fósforo, se procedió a calcular el costo de reparación del suelo/ha, desde la perspectiva ambiental causado por la reconversión de cacao arriba a cacao CCN51.

Los insumos necesarios para reparar el daño al suelo son: urea y fostato di-amónico. Las cantidades a aplicar son de 150 y 200 kg., respectivamente, por el tiempo de cinco años. Para la aplicación de estos insumos solamente se requerirán dos jornales al año, por el mismo tiempo. Los precios de mercado de los insumos en el mercado local son de USD $0,38 / \mathrm{kg}$ para la urea; $\mathrm{y}$, USD 0,60/kg para el DAP. El precio del jornal en la zona de la investigación es de USD 15,00 (Tabla 8).

Aplicando la fórmula de costo de reparación, una tasa de descuento $(r=12 \%)$, el costo de restauración estimado por hectárea, para el daño ambiental del recurso suelo es de USD 764,03 . Asumiendo, que esta situación se haya mantenido en toda la zona de Moraspungo, el daño total alcanza los USD 649,422 en las 850 ha de cacao CCN51 que existen sembradas actualmente en la UOPAM, de la siguiente manera: 
Tabla 2. Costo de reparación del daño ambiental en el recurso suelo, 2018 en la zona de Moraspungo, Provincia Cotopaxi, 2018.

\begin{tabular}{|c|c|c|c|c|c|c|c|c|}
\hline \multirow{4}{*}{\multicolumn{2}{|c|}{ Insumos ( $m=2)$}} & \multirow{5}{*}{ Unidad } & \multirow{5}{*}{$\begin{array}{c}\text { Precio } \\
\text { unitario } \\
\left(p_{i}\right)\end{array}$} & \multicolumn{5}{|c|}{ Recursos afectados $(n=1)$} \\
\hline & & & & \multicolumn{5}{|c|}{ Cantidad del insumo (q) } \\
\hline & & & & \multirow{2}{*}{$\begin{array}{c}\text { Año } 1 \\
t=0\end{array}$} & \multirow{2}{*}{$\begin{array}{c}\text { Año } 2 \\
t=1\end{array}$} & \multirow{2}{*}{$\begin{array}{c}\text { Año } 3 \\
t=2 \\
\end{array}$} & \multirow{2}{*}{$\begin{array}{l}\text { Año } 4 \\
t=3\end{array}$} & \multirow{2}{*}{$\begin{array}{l}\text { Año } 5 \\
t=4\end{array}$} \\
\hline & & & & & & & & \\
\hline & & & & Suelo & Suelo & Suelo & Suelo & Suelo \\
\hline & & & & $j=1$ & $j=1$ & $j=1$ & $j=1$ & $j=1$ \\
\hline Mano de obra & $i=1$ & jornal & 15 & 2 & 2 & 2 & 2 & 2 \\
\hline Urea $(46 \% \mathrm{~N})$ & $i=2$ & $\mathrm{~kg}$ & 0,38 & 150 & 150 & 150 & 150 & 150 \\
\hline DAP (18-46-00) & $i=3$ & $\mathrm{~kg}$ & 0,60 & 200 & 150 & 100 & 100 & 100 \\
\hline $\begin{array}{l}C R / h a= \\
150)+(0,6 \\
0.12)^{-2}+ \\
150)+(0,6\end{array}$ & $\begin{array}{l}60 * 15 \\
{[(15 *} \\
50 * 10\end{array}$ & $\begin{array}{l}+(0,38 \\
0)](1+0.1 \\
+(0,38 \\
0)](1+0 .\end{array}$ & $\begin{array}{l}2)^{-1}+[( \\
* 150)+( \\
12)^{-4}\end{array}$ & $\begin{array}{l}, 60 * 200 \\
15 * 2)+ \\
, 60 * 100\end{array}$ & $\begin{array}{l})](1+0 \\
(0,38 * 1 \\
)](1+0\end{array}$ & $\begin{array}{l}12)^{0}+[( \\
50)+(0, \\
12)^{-3}+\end{array}$ & $\begin{array}{l}5 * 2)+( \\
0 * 100)] \\
{[(15 * 2)}\end{array}$ & $\begin{array}{l}0,38 * \\
(1+ \\
-(0,38 *\end{array}$ \\
\hline
\end{tabular}

Fuente: Elaboración propia.

El uso intensivo e indiscriminado de los bienes ambientales, en especial del suelo, está generando un fuerte impacto ambiental negativo en el recurso, de tal forma que, si no se propende por su protección y conservación, puede llegarse a perder sus características y por ende su capacidad para ser empleado dentro de otros procesos productivos posteriores o como soporte para actividades de ocio y recreo, entre otras. En ese sentido, es importante insertar planes de gestión ambiental en nuestras organizaciones agrícolas para proteger y conservar los recursos ambientales (Silva y Correa 2010).

En un estudio relacionado con la evaluación económica sobre el rendimiento hídrico en una cuenca de bosque nativo y otra cuenca de Pinus radiata, determinaron que este parámetro se redujo de manera acelerada en la cuenca plantada con Pinus, con respecto a la cuenca con bosque nativo. La valoración económica determinó una pérdida en el bienestar resultante de un cambio de uso del suelo, por efecto de la siembra de diferentes especies de árboles (Oyarzún y Núñez 2015).

Por otro lado, esta investigación estimó el valor económico del daño ambiental en el recurso suelo, provocado por el cambio de la variedad de siembra de cacao entre 2011 y 2018 en 
la zona de Moraspungo. Los resultados muestran una pérdida significativa de los elementos nitrógeno y fósforo. Estos dos macroelementos del suelo fueron extraídos por la exigencia de nutrientes del cacao CCN51 sembrado en monocultivo con una población de alrededor de 1000 árboles por hectárea. La reposición de los elementos los nutrientes perdidos, causados por la siembra de CCN51, indican que al menos se necesitan cinco años continuos de aplicación de $\mathrm{N}$ y $\mathrm{P}$ en las cantidades establecidas anteriormente. Por lo tanto, se estimó que el costo de reparación para reponer el daño ambiental en el suelo, se requiere al menos USD 764,03 por hectárea y USD 649422 en las 850 has de cacao CCN51 que existen en la actualidad en toda la zona de Moraspungo.

A pesar de que la literatura consultada sobre la valoración económica del daño ambiental del suelo en el Ecuador no es abundante, un estudio metodológico realizado en Costa Rica por el Instituto de Políticas para la Sostenibilidad (Barrantes, Gerardo; Di Mare, María 2001), permitió estimar económicamente el costo de reparación de un recurso dañado por efecto de las actividades agropecuarias.

Si la externalidad negativa es remediada, el daño ambiental no desaparece, pues el impacto ambiental, se mantendrá aun cuando los recursos invertidos para la remediación se dirijan a combatir las causas que provocaron ese daño; por ejemplo, incorporando al suelo algún insumo que lo descontamine (Delacámara 2008).

Diversos autores analizan el tema del daño ambiental de suelo por el uso de sustancias químicas. (Silva y Correa 2010), destacan el hecho de que, aunque las actividades económicas, especialmente la agricultura, vienen sustituyendo el uso de sustancias como plaguicidas y fertilizantes por otras menos contaminantes, el ritmo de sustitución está por debajo del crecimiento agrícola, por lo cual se presenta un des-balance que genera un uso intensivo del recurso suelo por parte de las sustancias químicas mencionadas. Igualmente, otras actividades productivas también realizan un uso intensivo de este recurso, situación que está conduciendo a una degradación creciente del suelo, así como a una pérdida irrecuperable del mismo.

La aplicación de agroquímicos (pesticidas y plaguicidas especialmente), ha demostrado que reduce la capacidad microbiana en el suelo, pérdida de biomasa, muerte de especies vegetales o enfermedad de las personas que están en contacto con estos productos tóxicos (Silva y Correa 2009). 
En Argentina, un estudio sobre las cuestiones económicas ambientales de las transformaciones agrícolas plantea que el monocultivo de la soya afecta notablemente los suelos, intensifica el uso de pesticidas y fertilizantes, y facilitará el desplazamiento de otras alternativas productivas más amigables con el medio ambiente (Pengue 2009).

Finalmente, bajo las consideraciones realizadas en este estudio, la hipótesis se comprueba en tanto y en cuanto, el sacrificio por mantener inalterable la forma convencional de cultivar cacao arriba, ha significado que los productores, por un lado, renuncien a aumentar sus ingresos monetarios por no sembrar cacao CCN51, y por otro, han contribuido a la reducción del daño ambiental del recurso suelo, en la parroquia Moraspungo.

\section{CONCLUSIONES}

El costo de reparación del daño ambiental provocado en el recurso suelo desde el año 2011 hasta el 2017 fue de USD 764,00/ha y el daño total de la zona de Moraspungo se estimó en USD 650,000,00/ha aproximadamente.

Aproximadamente existen sembradas en el Ecuador unas 80,000 ha. sembradas de cacao CCN51, por lo que se podría inferir ceteris paribus el costo total para reparar la pérdida de $\mathrm{N}$ y $\mathrm{P}$ en el suelo por efecto de esta variedad sería de USD 61,120,000,00.

\section{REFERENCIAS BIBLIOGRÁFICA}

ANECACAO, Asociación Nacional de Exportadores de Cacao. (17 de octubre de 2012) www.anecacao.com. Consultado el 9 de abril de 2018. Recuperado de www.anecacao.com/index.php/es/estadisticas/estadisticas-actuales.html

Barrantes, G. y Di Mare, M. (2001). Metodogía para la evaluación económica de daños ambientales en Costa Rica. Instituto de políticas para la sostenibilidad, p. 53.

Barzeb, R. (2002). Guía metodológica de valoración económica de bienes, servicios e impactos ambientales. Managua: Corredor Biológico Mesoamericano.

Castellanos, M. (2007). Introducción a la problemática de la valoración económica ambiental. La Habana: Editorial Academia. 
Cristeche, E. y Penna, J (2008). Métodos de valoración económica de los servicios ambientales. Estudios socioeconómicos de la sustentabilidad de los sistemas de producción y recursos naturales, p. 7.

Delacámara, G. (2008). Guía para decisores. Análisis económico de externalidades ambientales. Santiago de Chile: Naciones Unidas.

Dixon, J. y Pagiola, S. (1998). «Análisis económico y evaluación ambiental.» Enviromental assessment sourcebook, p. 8.

Domínguez, M. (2004). «El papel de la fisiocracia en nuestros dias: una reflexión sobre el análisis económico de los recursos naturales y el medio ambiente.» Revista Galega de Economía.

Federación Nacional de Cacaoteros, FEDECACAO. (19 de octubre de 2012). Concultado 17 de febrero de 2010. Recueperado de http://www.fedecacao.com.co/cw/ca/doctecnicos/fedecacao-dt-guia-ambientalcacao-2010-febrero-17.pdf.

Gómez, C., Barrón, K. y Moreno, L. (2011). Crecimiento económico y medio ambiente en México. Trimestre económico, p. 547.

Instituto de Política para la Sostenibilidad. (28 de mayo de 2018). Metodogía para la evaluación económica de daños ambientales en Costa Rica. Recuperado de http://www.ips.or.cr. s.f. www.ips.or.cr/publicaciones_web.htm?idcuerpo=477.

Labandeira, X., León, C. y Vásquez, M. J. (2007). Economía ambiental. México: Pearson educacación S.A.

Morán, R. (2001). Aproximaciones al costo económico de impactos ambientales en la minería. Ambiente y desarrollo, 2001, pp. 59-67.

Morillo, M. (2001). Rentabilidad financiera y reducción de costos. Actualidad contable FACES, pp. 35-48.

Müggenburg, M. C., y Pérez, I. (2007). «Tipos de estudio en el enfoque de investigación cuantitativa .» Enfermería Universitaria, pp. 35-38.

Muñoz, M. (2001). Perfil de la Factibilidad. Quito: Alba Yala.

Organización de las Naciones Unidas para la Alimentación y Agricultura - FAO. (2011). Manual para la valoración social de: impactos y daños ambientales de actividades agrícolas. Caracas. 
Osorio, J., y Correa, F. (2004). Valoración económica de costos ambientales: marco conceptual y métodos de estimación. Semestre económico, pp. 163-164.

Oyarzún, C., Nahuelhual, L., y Núñez, D. (2015). Los servicios ecosistémicos del bosque templado lluvioso: producción de agua y su valoración económica. Ambiente y desarrollo de CIPMA, pp. 88-97.

Pengue, W. (2009). Cuestiones económico-ambientales de las transformaciones agrícolas en las Pampas. Revista latinoamericana de economía, pp. 137-161.

Peña, M. (1989). Reparación y valoración económica de los daños causados al medio ambiente. Medio ambiente\&derecho, pp. 3-4.

Peña, M. (2013). Daño ambiental y prescripción.» Revista Judicial, p. 118.

Pérez, J. (3 de Febrero de 2018). Enciclopedia de conceptos. Consultado el 2 de agosto de 2018. Recuperado de https://concepto.de/medio-ambiente/

Silva, S., y Correa, F. (2009). Análisis de la contaminación del suelo: revisión de la normativa y posibilidades de regulación económica. Semestre económico, pp. 1334.

Silva, S., y Correa, F. (2010). Valoración económica del suelo y gestión ambiental: aplicación en empresas florícolas colombianas. Revista Facultad de Ciencias Económicas: investigación y reflexión, p. 265.

Unibertsitatea, H., y Cano, N. (2001). Teoría de las tres dimensiones de desarrollo sostenible. Ecosistemas, pp. 1-2.

Vargas, A., y Reyes, M. (2011). Incentivos económicos para la conservación de áreas naturales. Una revisón de la literatura. Lecturas de economía, pp. 151-170. 